\title{
Comunicación
}

\section{Reporte de Caso: Rabdomiosarcoma Embrional en un Bulldog Inglés}

\author{
Case Report: Embryonal Rhabdomyosarcoma in an English Bulldog \\ Jessenia Julieth Rojas Hurtado ${ }^{1}$, Dunia Yisela Trujillo Piso ${ }^{2,4}$, \\ Carlos Augusto Rengifo Valbuena ${ }^{3}$
}

\section{Resumen}

Este artículo describe el caso clínico de un canino Bulldog Inglés, hembra, de 10 meses de edad, llevado a consulta por presentar un nódulo superficial y de crecimiento acelerado en la zona abdominal izquierda. Al examen físico se encuentra una masa sésil, de aproximadamente $2 \times 3 \mathrm{~cm}$, en la piel del flanco izquierdo, lateral a la segunda glándula mamaria, no dolorosa. La paciente fue sometida a biopsia escisional para retirar la masa en su totalidad. El estudio histopatológico determinó rabdomiosarcoma embrional, una patología poco frecuente en caninos, tratada con éxito y sin recidiva reportada en los seis meses posteriores de su remoción.

Palabras clave: rabdomiosarcoma embrional, histopatología, Bulldog inglés, canino

\section{Abstract}

This paper describes the clinical case of a female English Bulldog canine, 10 months old, that was brought to consultation due to the presence of a superficial and accelerated growth node in the left abdominal area. The physical exam showed a sessile mass, approximately $2 \times 3 \mathrm{~cm}$, located on the skin of the left flank, close to the second mammary gland, and painless. The patient was subjected to an excisional biopsy to remove the entire mass. The histopathological study showed an embryonal rhabdomyosarcoma, a rare disease in dogs. The removal of the neoplasm was successful and without reported recurrence in the six months after its removal.

Key words: rhabdomyosarcoma, histopathology, English Bulldog, canine

\footnotetext{
${ }^{1}$ Clínica Veterinaria Punto Mascotas, Montenegro Quindío, Colombia

${ }^{2}$ Grupo de Investigación IMPRONTA, Universidad Cooperativa de Colombia, Ibagué, Colombia

${ }^{3}$ Facultad de Ciencias, Universidad del Tolima, Ibagué, Colombia

${ }^{4}$ E-mail: dunia.trujillop@campusucc.edu.co
}

Recibido: 10 de junio de 2016

Aceptado para publicación: 14 de octubre de 2016 


\section{INTRODUCCIÓN}

En la rutina de la clínica veterinaria es común la presentación de diferentes tipos de neoplasias, dentro de las cuales raramente se reportan los rabdomiosarcomas (Hulland, 1990); tumores malignos provenientes de células musculares estriadas (Moulton, 1978), de común presentación en la especie humana, pero de baja incidencia en animales. Entre estos últimos, se reportan mayormente en caninos, seguidos de equinos y felinos (Valentine y McGavin, 2007).

La tasa de incidencia del rabdomiosarcoma en caninos es menos del $1 \%$ dentro de todas las neoplasias presentadas en esta especie (Ginel et al., 2002), y dentro de los sarcomas de tejidos blandos representa el 3.8\% (Silveira et al., 2007). Moulton (1978) y Cooper y Valentine (2002) los han descrito principalmente en animales jóvenes, de menos de dos años. Asimismo, se les han encontrado en cuello, lengua, miocardio y tracto urinario (Cooper y Valentine, 2002; Valentine y McGavin, 2007), así como en la región maxilofacial (Nakaichi et al., 2007), laringe (Block et al., 1995), región parameníngea (Illanes, 2002) y región perianal (Ueno et al., 2002).

Los rabdomiosarcomas tienen su origen en el músculo esquelético, pero pueden desarrollarse en cualquier parte del organismo, incluso en regiones donde el músculo estriado no está presente, como es el caso de faringe, vejiga urinaria, útero y cérvix (Moulton, 1978; Van Vleet, 2000; Cooper y Valentine, 2002).

El rabdomiosarcoma es una neoplasia maligna, típicamente agresiva e invasiva, con capacidad de metástasis (Akkoc et al., 2006; Bae et al., 2007) y cuyas manifestaciones clínicas están asociadas al lugar de aparición (Moulton, 1978); por tanto, su diagnóstico es exclusivamente histopatológico. Técnicas de inmunohistoquímica, microscopía electrónica y el análisis inmunohistoquímico con anticuerpos monoclonales para la mioglobina y la miosina ayudan en el diagnóstico definitivo (Kobayashi et al., 2004). No obstante, la técnica diagnóstica más precisa para la confirmación del diagnóstico de rabdomiosarcomas es la detección de proteínas específicas de las células musculares mediante inmunohistoquímica (Sardon et al., 2012).

El potencial de metástasis y el pronóstico de perros con rabdomiosarcoma no ha sido determinado, debido a que esta condición es raramente diagnosticada e incluso raramente tratada (Hulland, 1990; Parham, 2001; Liptak y Forrest, 2007; Inga et al., 2013); sin embargo, su clasificación histológica, estadificación y localización sugieren que los del tipo botroide son de pronóstico favorable y los de peor pronóstico son los tipos alveolar y pleomórfico (Crist et al., 1995; Raney et al., 2002; Breneman et al., 2003).

Los rabdomiosarcomas, según sus características histológicas, se clasifican en embrional, botrioide, pleomorficas y alveolar (Moulton, 1978; Meuten, 2002). El tipo embrional es el más común en caninos, presentando tres subtipos: El rabdomioblástico, compuesto por células redondas a poligonales o rabdomioblastos, mezcladas con pequeñas células; el 'Spindle cell', caracterizado por la presencia de filas de células fusiformes grandes; y el miotubular, cuya histología es similar al tumor de células fusiformes, descrito en humanos, y compuesto por células similares a miocitos primitivos, denominados como miotubos (Cooper y Valentine, 2002; Caserto, 2013).

El rabdomiosarcoma embrional miotubular es la variante más común en caninos y, junto al rabdomiosarcoma botrioide, es el más frecuente en el tracto urinario, mientras que el de células redondas usualmente surge en otras localizaciones (Yanoff et al., 1996; Cooper y Valentine, 2002; Kobayashi et al., 2004; Susuki et al., 2006; Bae et al., 2007; Nakaichi et al., 2007). 
El tratamiento del rabdomiosarcoma depende de la histología de la neoplasia, edad del paciente, de la localización y tamaño, y de la etapa preoperatoria (Morkawa, 2005; Inga et al., 2013). Los pacientes con rabdomiosarcomas requieren un tratamiento de múltiple modalidad, con quimioterapia sistémica, conjuntamente con cirugía y radioterapia (Ardilla, 2005; Inga et al., 2013).

Si bien en caninos existen únicamente dos reportes de rabdomiomas cutáneos (Cooper y Valentine, 2002), reportes de rabdomiosarcomas de tipo embrional cutáneo son tan escasos que solo se conoce un relato reciente en un canino de raza Pointer Inglés (Avallone et al., 2010). En el presente estudio se reporta un caso de rabdomiosarcoma embrional cutáneo en un canino joven de raza Bulldog Inglés.

\section{Caso Clínico}

Un paciente canino Bulldog Inglés, hembra, de 10 meses de edad y con $19.8 \mathrm{~kg}$ de peso corporal, ingresó al centro veterinario Punto Mascota, ubicado en Montenegro, Quindío, Colombia, por un aparente nódulo en piel de la región abdominal izquierda. La propietaria lo reportó como una picadura de insecto que se hizo extensa en un periodo no mayor de ocho días. Previamente, la paciente había presentado alteraciones dermatológicas caracterizadas por alopecia en tren posterior, con resultado negativo para hongos (hidróxido de potasio, $\mathrm{KOH}$ ) y sin presencia de ácaros, que fue tratada con Uniclav ${ }^{\circledR}$ (amoxicilina + ácido clavulánico), en tabletas de $500 \mathrm{mg}$ a una posología de 15 $\mathrm{mg} / \mathrm{kg}$ /día durante 10 días, y clorhexidina tópica, por ocho semanas continuas con resolución de la patología.

\section{Examen Clínico}

Las constantes fisiológicas se encontraron dentro del rango de normalidad. Se en- contró una masa de aproximadamente $2 \mathrm{~cm}$ de largo por $3 \mathrm{~cm}$ de ancho, sésil, regular e hiperpigmentada en el tegumento de la zona abdominal izquierda, lateral a la segunda glándula mamaria torácica, sin dolor a la palpación. La masa no presentaba adherencias, por lo que se decide realizar biopsia escisional, para lo cual se solicitan exámenes prequirúrgicos (hemograma, perfil renal y hepático). Los resultados de estos exámenes no muestran alteración digna de nota y se programa a la paciente para el procedimiento quirúrgico.

\section{Biopsia}

La biopsia fue realizada utilizando el siguiente protocolo: medicación preanestésica con xilacina $0.5 \mathrm{mg} / \mathrm{kg}$ y tramadol $4 \mathrm{mg} / \mathrm{kg}$, inducción anestésica con propofol $5 \mathrm{mg} / \mathrm{kg}$ y mantenimiento de anestesia con propofol a 3 $\mathrm{mg} / \mathrm{kg}$. Además, se realizó infiltración local con lidocaína. Se remueve la masa dejando un margen quirúrgico de $2 \mathrm{~cm}$ alrededor de la neoplasia. El cierre de la piel se realiza con patrón subcuticular y puntos simples discontinuos. Una muestra de la neoplasia se envía en formol al $10 \%$ para estudio histopatológico.

\section{Estudio Histopatológico}

Los resultados del examen de histopatología revelaron crecimiento celular expansivo que establece proceso neoplásico carente de cápsula. El colágeno aparece desplazado y se observan dos patrones ricos en figuras mitóticas, uno sólido de células anisocarióticas y pleomórficas con procesos de contacto citoplásmico, agrupadas sutilmente por delgados septos estromales y un segundo patrón de ordenamiento columnar (rabdomioblastos). En la zona central de patrón sólido, son frecuentes las células que adoptan forma de cinta y ubican sus núcleos a la periferia de la misma (Figura 1), hallazgos que permiten diagnosticar rabdomio-sarcoma embrional. 

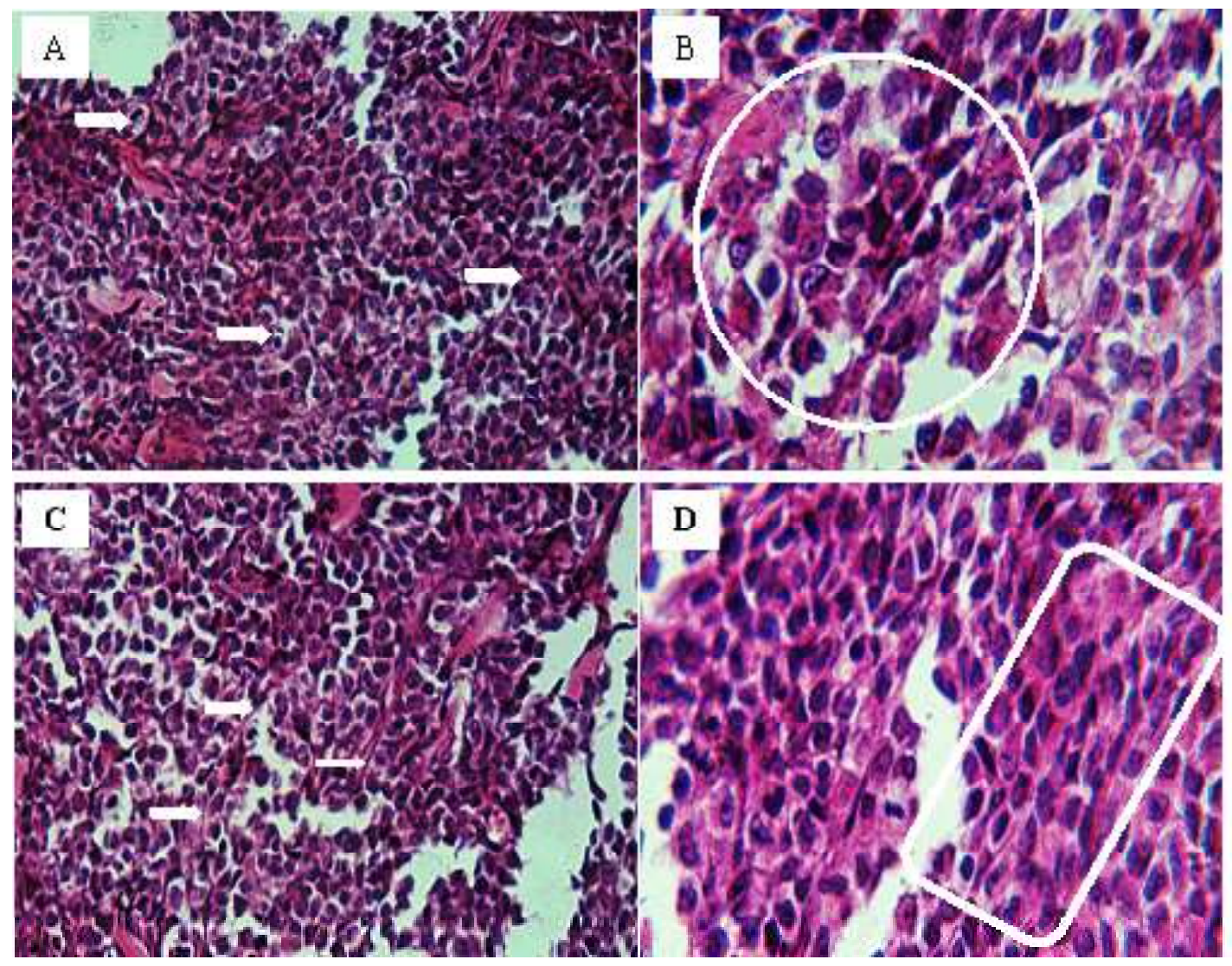

Figura 1. Cortes histológicos de una biopsia de masa tumoral cutánea en canino Bulldog Inglés, hembra, de 10 meses de edad (Montenegro, Quindío, Colombia). A. Figuras mitóticas. B. Pleomorfismo y anisocariosis (círculo blanco). C. Septos estromales. D. Ordenamiento columnar - rabdomioblastos

\section{Tratamiento}

No se realizó tratamiento médico complementario con quimioterapia debido al diagnóstico histopatológico. Cabe resaltar que no ha habido reincidencia en la paciente luego de seis meses de la extirpación de la masa tumoral.

\section{Discusión}

El rabdomiosarcoma es un tumor derivado de las células precursoras del músculo esquelético, clasificado como embrional, alveolar o pleomórfico. En caninos, el tipo embrional es el más común (Cooper y Valentine, 2002).
El caso de rabdomiosarcoma embrional subcutáneo reportado por Avallone (2010) demuestra el alto potencial invasivo de este subtipo de neoplasia en caninos, pues se le encontró metástasis en ganglios linfáticos regionales y hubo recurrencia seis meses después de realizar la escisión y la quimioterapia. Asimismo, se dispone de reportes similares como el de Yhee et al. (2008), quienes observaron osteólisis de la escápula e invasión al pulmón en un perro de ocho meses de edad, y el de Illanes (2002), quien encontró rabdomiosarcoma de tipo embrionario en región parameningea con consecuente denervación y atrofia de los músculos masticatorios, además de metástasis al hígado. No obstante, Yanoff et al. (1996) reportaron el caso no agresivo de esta neoplasia en la 
tráquea de un paciente de cuatro meses, que no presentó recidiva o metástasis luego de extracción quirúrgica del tumor. El diagnóstico y el tratamiento quirúrgico temprano pueden ser la clave en la mejoría de estos pacientes, como pudo haber sido el caso de Yanoff et al. (1996) y el del presente estudio.

La edad y la raza han sido otros factores a tener en cuenta para entender el comportamiento de los rabdomiosarcomas. La edad más frecuente de presentación de esta neoplasia en caninos es de menos de dos años (Moulton, 1978; Cooper y Valentine, 2002; Valentine y McGavin, 2007), aunque un estudio reciente sugiere que, específicamente, el rabdomiosarcoma de tipo embrional ocurre más comúnmente en perros adultos de 6 a 10 años de edad (Inga et al., 2013), en contraposición al caso de humanos, donde se presenta principalmente en niños menores de 10 años (Weis y Goldblumb, 2009). En el tejido subcutáneo, el paciente reportado por Avallone et al. (2010) tenía 11 años y el del presente relato tenía 10 meses de edad. Se requiere de reportes adicionales para determinar si la edad del paciente es un factor de importancia para la presentación de estas neoplasias.

El rabdomiosarcoma acomete con mayor frecuencia a caninos de razas puras (Inga et al., 2013), pero la relación entre la raza y el tipo de rabdomiosarcoma no ha sido debidamente estudiado. Solo se ha establecido una relación entre el rabdomiosarcoma embrionario botrioide con caninos de razas grandes como San Bernardo (Bae et al., 2007).

El rabdomiosarcoma primario cutáneo es frecuentemente mal diagnosticado o pobremente diferenciado de otras neoplasias con comportamiento similar (Villamil et al., 2011), de allí que para su correcto diagnóstico se requiere de técnicas de inmunohistoquímica (Vos et al., 1993; Wachtel, 2006; Avallone, 2010) o histopatología (Yhee et al., 2008), especialmente con el uso de miosina y mioglobina como marcadores (Kobayashi et al., 2004). Una posibilidad de confusión es con el sarcoma anaplásico (Caserto, 2013).

En los pacientes con rabdomiosarcoma se recomienda realizar remoción quirúrgica, quimioterapia e incluso radioterapia (Avallone et al., 2013), con lo cual se busca mejorar la calidad de vida del paciente (Scott et al., 2015); sin embargo, la posibilidad de recidiva y metástasis es evidente (Avallone et al., 2013) y las consecuencias de esta dependerán de la ubicación primaria del tumor (Gerber et al., 2009).

\section{Literatura Citada}

1. Akkoc A, Ozyigit MO, Yilmaz R. Alasonyalilar A, Cangul IT. 2006. Cardiac metastasising rhabdomyosarcoma in a Great Dane. Vet Rec 158: 803804.

2. Ardilla F. 2005. Tratamiento quimioterapéutico convencional en rabdomiosarcoma embrionario. Resistencia a drogas y modulación antigénica. Tesis Doctoral. Granada, España: Facultad de Medicina, Universidad de Granada. 197 p.

3. Avallone G, Pinto da Cunha $N$, Palmieri C, Della Salda L, Stefanello D, Roccabianca P, Caniatti M. 2010. Subcutaneous embryonal rhabdomyosarcoma in a dog: cytologic, immunocytochemical, histologic, and ultrastructural features. Vet Clin Pathol 39: 499-504. doi: 10.1111/j.1939-165X.2010.00271.x

4. Bae IH, Kim Y, Pakhrin B, You MH, Hwang CY, Kim JH, Kim DY. 2007. Genitourinary rhabdomyosarcoma with systemic metastasis in a young dog. Vet Pathol 44: 518-520. doi: 10.1354/vp.44-4518

5. Block G, Clarke K, Salisbury S, DeNicola D. 1995. Total laryngectomy and permanent tracheostomy for treatment of laryngeal rhabdomyosarcoma in a dog. J Am Anim Hosp Assoc 31:510-513. doi: 10.5326/15473317-31$6-510$ 
6. Breneman JC, Lyden E, Pappo AS, Link MP, Anderson JR, Parham DM, Qualman SJ, et al. 2003. Prognostic factors and clinical outcomes in children and adolescents with metastatic rhabdomyosarcoma - a report from the Intergroup Rhabdomyosarcoma Study IV. J Clin Oncol 21: 78-84. doi: 10.1200/JCO.2003.06.129

7. Caserto BG. 2013. A comparative review of canine and human rhabdomyosarcoma with emphasis on classification and pathogenesis. Vet Pathol 50: 806-826. doi: 10.1177/ 0300985813476069

8. Cooper BJ, Valentine BA. 2002. Tumors of muscle. In: Meuten DJ. Tumors in domestic animals. $4^{\text {th }}$ ed. Iowa, USA: Iowa State Press. p 319-363.

9. Crist W, Gehan E, Ragab A, Dickman P, Donaldson S, Fryer C, Hermann $J$, et al. 1995. The Third Intergroup Rhabdomyosarcoma Study. J Clin Oncol 13:610-630.

10. Gerber K, Rees P. 2009. Urinary bladder botryoid rhabdomyosarcoma with widespread metastases in an 8month-old Labrador cross dog. J S Afr Vet Ass 80: 199-203.

11. Ginel PJ, Martin de las Mulas J, Lucena R, Millán Y, Novales M. 2002. Skeletal muscle rhabdomyosarcoma in a dog. Vet Rec 151: 736-738.

12. Hulland T. 1990. Tumors of the muscle. In: Moulton JE (ed). Tumors in domestic animals. $3^{\text {rd }}$ ed. Los Angeles, USA: Univ California. pp 93-100.

13. Illanes $O G$ 2002. Juvenile parameningeal rhabdomyosarcoma in a dog causing unilateral denervation atrophy of masticatory muscles. J Comp Pathol 126: 303-307. doi: 10.1053/jcpa.2001.0546

14. Inga ER, Sandoval N, Perales R, Chavera A. 2013. Frecuencia de rabdomiosarcoma canino en el Laboratorio de Histopatología Veterinaria de la Universidad Nacional Mayor de San Marcos (Periodo 2000-2008). Rev Inv Vet Peru 24: 293-299. doi: 10.15381/ rivep.v24i3.2577
15. Kobayashi M, Sakai H, Hirata A, Yonemaru K, Yanai T, Watanabe K, Yamazoe K, et al. 2004. Expression of myogenic regulating factors, myogenin and MyoD, in two canine botryoid rhabdomyosarcomas. Vet Pathol 41: 275277. doi: $10.1354 / v p .41-3-275$

16. Liptak JM, Forrest LJ. 2007. Soft tissue sarcomas. In: Withrow SJ, Vail DV (eds). Small animal clinical oncology. $4^{\text {th }}$ Ed. USA: Elsevier. p 425-454.

17. Meuten DJ. 2002. Tumors in domestic animals. $4^{\text {th }}$ ed. USA: Wiley-Blackwell. $800 \mathrm{p}$.

18. Morkawa Y. 2005. Childhood rhabdomyosarcoma. Nippon Geka Gakkai Zasshi 106: 431-436.

19. Moulton JE. 1978. Tumors in domestic animals. $2^{\text {nd }}$ ed. USA: University of California Press. 480 p.

20. Nakaichi M, Itamoto K, Hasegawa K, Morimoto M, Hayashi T, Une S, Taura Y, Tanaka K. 2007. Maxillofacial rhabdomyosarcoma in the canine maxilofacial area. J Vet Med Sci 69: 6567.

21. Parham DM. 2001. Pathologic classification of rhabdomyosarcomas and correlations with molecular studies. Mod Patholol 14: 506-514.

22. Raney RB, Meza J, Aderson JR, Fryer CJ, Donaldson SS, Breneman JC, Fitzgerald TJ, et al. 2002. Treatment of children and adolescents with localized parameningeal sarcoma: experience of the Intergroup Rhabdomyosarcoma Study Group protocols IRS-II through IV, 1978-1977. Med Pediatr Oncol 38: 22-32. doi: 10.1002/ mpo. 1259

23. Sardon D, Herrozo I, Vasquez F. 2012. Rabdomiosarcoma embrionario en un perro. Biociencias 8.9 p. [Internet]. Disponible en: https://dialnet.unirioja.es/ servlet/oaiart?codigo $=5465798$

24. Scott EM, Teixeira LB, Flanders DJ, Dubielzig RR, McLellan GJ. 2015. Canine orbital rhabdomyosarcoma: a report of 18 cases. Vet Ophthalmol 19: 130-137. doi: 10.1111/vop.12270 
25. Silveira MF, Guim TN, Spader M, Schuch I, Conrado de Oliveira G, Bonel-Raposo J, Fernandes CG. 2007. Estudo retrospectivo de 63 casos de sarcomas de tecido mole no período de 1980-2005. Rev Cient Fac Agr Eliseu Maciel-UFPel 2: 18-21.

26. Susuki K, Nakatani K, Shibuya H, Sato T. 2006. Vaginal rhabdomyosarcoma in a dog. Vet Pathol 43: 186188. doi: 10.1354/vp.43-2-186

27. Ueno H, Kadosawa T, Isomura $H$, Okada Y, Ochiai K, Umemura T, Okumura M, Fujinaga T. 2002. Perianal rhabdomyosarcoma in a dog. J Small Anim Pract 43: 217-220. doi: 10.1111/j.1748-5827.2002.tb00061.x

28. Valentine BA, McGavin MD. 2007. Skeletal muscle. In: McGavin M, Zachary JF (eds). Pathologic basis of veterinary disease. $4^{\text {th }}$ ed. St Louis, USA: Mosby Elsevier. p 973-1040.

29. Van Vleet JF. 2000. Músculo esquelético. In: Jones TC, Hunt RD, King NW (eds). Patologia Veterinária. $6^{\mathrm{a}}$ ed. Brasil: Manole. p 887-911.

30. Villamil JA, Henry CJ, Bryan JN, Ellersieck M, Schultz L, Tyler JW, Hahn AW. 2011. Identification of the most common cutaneous neoplasms in dogs and evaluation of breed and age distributions for selected neoplasms. J Am Vet Med Assoc 239: 960-965. doi: 10.2460/javma.239.7.960

31. Vos JH, Borst GHA, Martin de las Mulas J, Ramaekers FC, van Mil FN, Molenbeek RF, Ivanyi D, van den Ingh TS. 1993. Rhabdomyosarcomas in young pigs in a swine breeding farm: a morphologic and immunohistochemical study. Vet Pathol 30: 271-279.

32. Wachtel M, Runge T, Leuschner I, Stegmaier S, Koscielniak E, Treuner $J$, Odermatt B, et al. 2006. Subtype and prognostic classification of rhabdomyosarcoma by immunohistochemistry. J Clin Oncol 24: 816-822. doi: 10.1200/JCO.2005.03.4934

33. Weiss SW, Goldblum JR. 2009. Enzinger y Weiss, tumores de partes blandas. España: Elsevier. 1268 p.

34. Yanoff SR, Fuentealba C, Boothe HW, Rogers KS. 1996. Tracheal defect and embryonal rabdomyosarcoma in a young dog. Can Vet J 37: 172-173.

35. Yhee JY, Kim DH, Hwang DN, Moon CT, Yu CH, Kim HY, Doster AR, Sur JH. 2008. Hematogenous metastasis of embryonal rhabdomyosarcoma originating from skeletal muscle in a young dog. J Vet Diagn Invest 20: 243-246. doi: $10.1177 / 104063870802000219$ 